\title{
Whole-genome analysis reveals the contribution of non-coding de novo transposon insertions to autism spectrum disorder
}

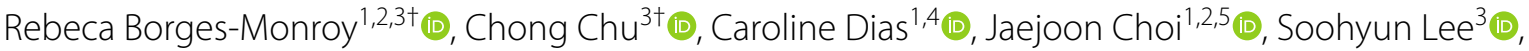

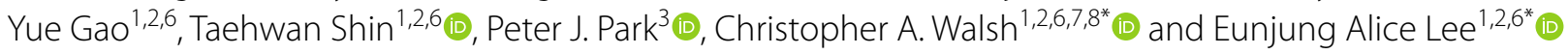

\begin{abstract}
Background: Retrotransposons have been implicated as causes of Mendelian disease, but their role in autism spectrum disorder (ASD) has not been systematically defined, because they are only called with adequate sensitivity from whole genome sequencing (WGS) data and a large enough cohort for this analysis has only recently become available.

Results: We analyzed WGS data from a cohort of 2288 ASD families from the Simons Simplex Collection by establishing a scalable computational pipeline for retrotransposon insertion detection. We report 86,154 polymorphic retrotransposon insertions-including $>60 \%$ not previously reported-and 158 de novo retrotransposition events. The overall burden of de novo events was similar between ASD individuals and unaffected siblings, with 1 de novo insertion per 29, 117, and 206 births for Alu, L1, and SVA respectively, and 1 de novo insertion per 21 births total. However, ASD cases showed more de novo L1 insertions than expected in ASD genes. Additionally, we observed exonic insertions in loss-of-function intolerant genes, including a likely pathogenic exonic insertion in CSDE1, only in ASD individuals.

Conclusions: These findings suggest a modest, but important, impact of intronic and exonic retrotransposon insertions in ASD, show the importance of WGS for their analysis, and highlight the utility of specific bioinformatic tools for high-throughput detection of retrotransposon insertions.
\end{abstract}

Keywords: Transposable elements, Retrotransposons, Autism spectrum disorder, de novo insertions, Polymorphic insertions, de novo rates, Alu, SVA, LINE-1, Neurobiology

\section{Background}

Retrotransposons contribute to genomic and transcriptomic variability in humans and cause a variety of human diseases [1]. Retrotransposons are a class of mobile DNA

*Correspondence: Christopher.Walsh@childrens.harvard.edu; ealice. lee@childrens.harvard.edu

${ }^{\dagger}$ Rebeca Borges-Monroy and Chong Chu contributed equally to this work. ${ }^{6}$ Department of Pediatrics, Harvard Medical School, MA, Boston, USA

Full list of author information is available at the end of the article elements that can copy themselves into RNA and insert themselves into new regions of the genome. This retrotransposition event is estimated to occur in one out of 20-40, 63-212, and 63-916 live births for Alu, LINE-1 (L1), and SVA elements respectively [2-4]. Transposable element insertions (TEIs) in both exons and non-coding regions can cause diseases by various mechanisms, including disrupting coding sequences, causing deletions, and altering RNA splicing, which can cause frameshifts and loss of function (LoF) [1]. To date, there are more 


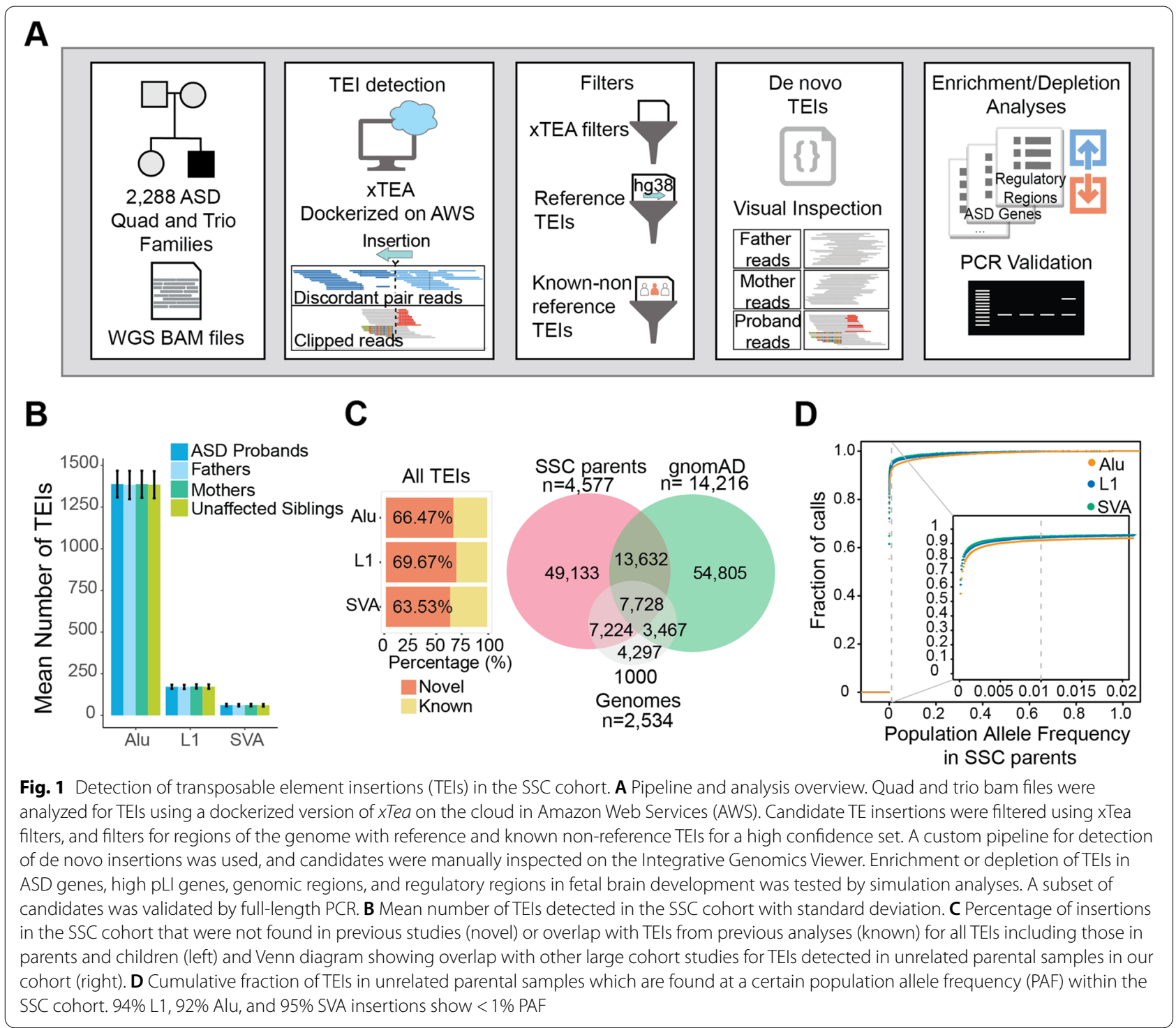

than 100 cases of TEIs causing diseases [1], including de novo insertions in developmental disorders [5]. A landmark study identified a deep intronic SVA insertion causing exon-trapping in a child with Batten disease, resulting in the development of a personalized antisense-oligonucleotide drug to fix the splicing defect [6]. Thus, the identification of TEs is important for increasing genetic diagnoses but also creates the promise of developing novel therapeutics for specific mutant alleles.

Autism Spectrum Disorder (ASD) is a heterogeneous developmental disorder characterized by communication deficits, impaired social interactions, and repetitive behaviors [7]. Although about $17-50 \%$ of the overall heritability of ASD reflects common variation at a population level, rare inherited and de novo copy-number variations and single nucleotide variations confer high risk to developing ASD, and drive ASD risk when present in individual children [8]. These rare variants are enriched in simplex families, where both parents are unaffected, with de novo copy-number variations and single nucleotide variations contributing to $30 \%$ of cases in the Simons Simplex Cohort (SSC) [9]. Although recent ASD studies have included TEIs [10-12], the smaller sample size and the low rates of de novo TEIs limited their analyses leaving the role of de novo TEIs in both exons and introns in ASD largely unknown. In this study, we sought to define the role of TEIs in ASD by analyzing the largest cohort of 2288 simplex families for de novo TEIs at whole genome resolution (Fig. 1A). 


\section{Results}

Most polymorphic insertions are rare and novel

We developed and implemented xTea [13], a scalable algorithm for detecting TEIs in whole-genome sequencing (WGS) data and demonstrated that the version of this tool used in our study has a high sensitivity, specificity, and comparable performance to MELT [14], the algorithm used to detect TEIs in gnomAD, as well as a better performance than Mobster [15] (Additional file 1: Fig. S1). Using $x$ Tea, we detected a total of 86,154 unique polymorphic TEIs $(68,643 \mathrm{Alu}$, $12,076 \mathrm{~L} 1$, and $5435 \mathrm{SVA}$ ) in the entire cohort (parents and children) (Additional file 1: Fig. S2A and Table S1). Each genome carried 1618 polymorphic TEIs on average (1385 Alu, $172 \mathrm{~L} 1$, and 61 SVA) comparable with previous analyses $[16,17]$, and the numbers were consistent across different family members (Fig. 1B and Additional file 1: Fig. S2A). We detected more Alu TEIs in African Americans, suggesting that TEI diversity is different in distinct populations $(\mathrm{F}(7)=970.8, p<2 \mathrm{e}-16$, one-way ANOVA) (Additional file 1: Fig. S3). 74\% of the overall TEIs detected (50,507 Alu, 9247 L1, 4273 SVA) were observed in either more than one individual in this cohort (71\%; 48,189 Alu, 8821 L1, 4021 SVA) or in previous studies (33\%; 23,018 Alu, 3663 L1, 1982 SVA) (Additional file 1: Fig. S2B), suggesting that most of these calls are bona fide. However, more than $60 \%$ of calls were novel and had not been detected before in gnomAD [18] or the 1000 genomes cohort [14] (Fig. 1C and Additional file 1: Fig. S4). In 4577 unrelated parental samples in our cohort, we detected 77,717 TEIs (dbVar "nstd203"), compared to the 79,632 insertions detected from 54,805 individuals in the gnomADSV cohort [18]. The majority of novel TEIs had the expected target-site duplication (TSD) size, and SVA and L1 novel TEIs display a similar TSD size distribution to known non-reference (KNR) TEIs (Additional file 1: Fig. S5A). The TSD size distribution of novel Alu TEIs with sufficient clipped and discordant read support on both breakpoints of the insertion, a polyA tail, and a TSD also resembles the TSD size distribution of KNR TEIs (Additional file 1: Fig. S5B). The high performance, especially high specificity ( $>75 \%$ for Alu, L1, and SVA at $\sim 40 \mathrm{X}$ sequencing coverage) of $x$ Tea (Additional file 1: Fig. S1) suggests that the majority of these novel TEIs are high confidence insertions. Additionally, insertions in our cohort had a higher overlap with previously published insertions from 2534 individuals in the 1000 genomes cohort [14] (Fig. 1C). The majority of parental TEIs were rare, for example, $>92 \%$ of TEIs having $<1 \%$ population allele frequency (PAF) within the analyzed cohort (Fig. 1D and Additional file 1: Fig.
S6), which is similar to previous findings for structural variants [18].

\section{ASD cases have more de novo insertions in ASD genes than expected}

We identified 158 de novo TEIs from all children (Additional file 2: Table S2). Previous studies have generally reported de novo TEI rates based on the number of insertions found in their cohort without accounting for detection sensitivity $[4,12]$. Multiple factors, including filtered regions, low sensitivity of the algorithm being used, or false negatives due to the sequencing methodology, result in an underestimate of true de novo rates. For example, TEI detection in Illumina short-read sequencing data is less sensitive than in long-read data, particularly for L1 TEIs [19, 20]. Therefore, we adjusted the observed de novo rates to account for sensitivity loss and to obtain precise estimates. We obtained adjusted de novo rates of 1 in 29 births for Alu (95\% CI 25-35), 1 in 117 births for L1 (95\% CI 85-168), and 1 in 206 births for SVA (95\% CI 134-336) (Fig. 2A and Additional file 1: Table S3).

We detected 62 de novo Alu insertions in ASD $(N=2286)$ and 57 in controls $(N=1857), 12$ de novo L1 insertions in ASD $(\mathrm{N}=2286)$ and 10 in controls $(N=1856)$, and 9 de novo SVA insertions in ASD $(N=2288)$ and 8 in controls $(N=1860)$ (Additional file 2: Table S2). We did not detect a difference in total de novo TEIs in ASD versus unaffected siblings (Fig. 2B) but unexpectedly observed a higher rate of intronic Alu insertions in controls $(p=0.003$, two-sided Fisher's Exact Test) (Fig. 2B). On the other hand, we observed a trend towards more exonic and intergenic Alu insertions in ASD than controls though not significant $(p=0.388$ for exonic insertions, $p=0.157$ for intergenic insertions, two-sided Fisher's Exact Test) (Fig. 2B) which leads to similar overall rates for de novo Alu insertions.

We detected de novo intronic L1 insertions in syndromic ASD genes curated by Simons Foundation Autism Research Initiative (SFARI) [21] only in ASD and not in controls, and the rate in ASD was higher than expected (empirical two-sided $p$-value using 10,000 permutation runs, $p=0.001$, q-value $=0.03$ ) (Fig. 3 ) (Table 1$)$. We also observed a trend for more de novo intronic L1 insertions in genes with high pLI scores indicating a high probability of loss of function intolerance [36] in ASD than expected (empirical two-sided $p$-value, $p=0.02$, q-value $>0.05$ ) (Additional file 1: Fig. S7). This approach is limited to observing an enrichment or depletion compared to a fully random model of TEIs, and we cannot account for factors that might influence the location of de novo insertions such as GC content and epigenetic context. However, these trends were also confirmed when 

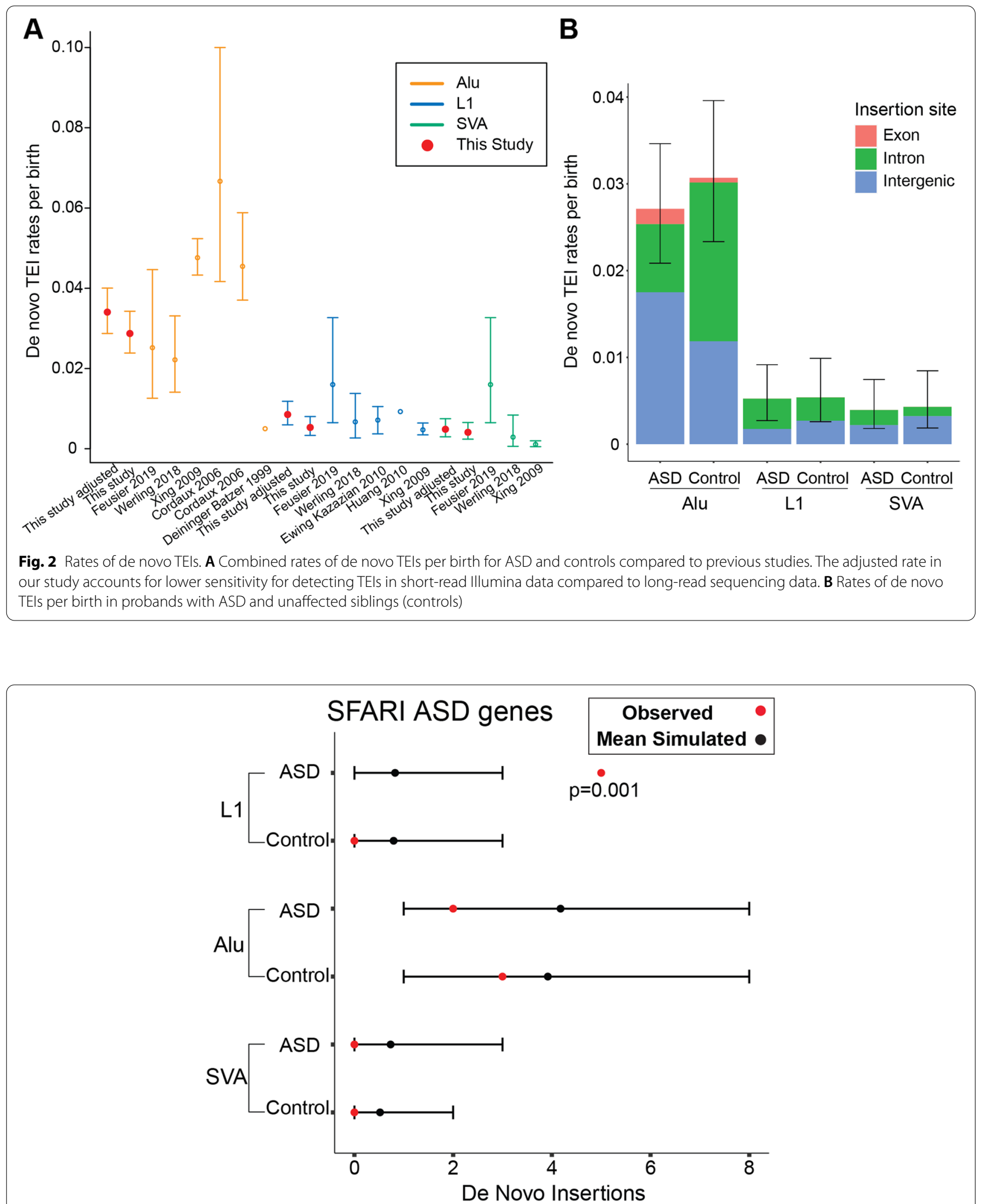

Fig. 3 Enrichment of de novo TEls in SFARI ASD genes. Observed numbers of de novo TEls in a list of complied ASD genes are marked by red dots. Black dots and lines represent mean numbers and 95\% confidence intervals of expected TEls based on 10,000 random simulations, respectively. More de novo L1 insertions in ASD genes than expected are observed in cases only 


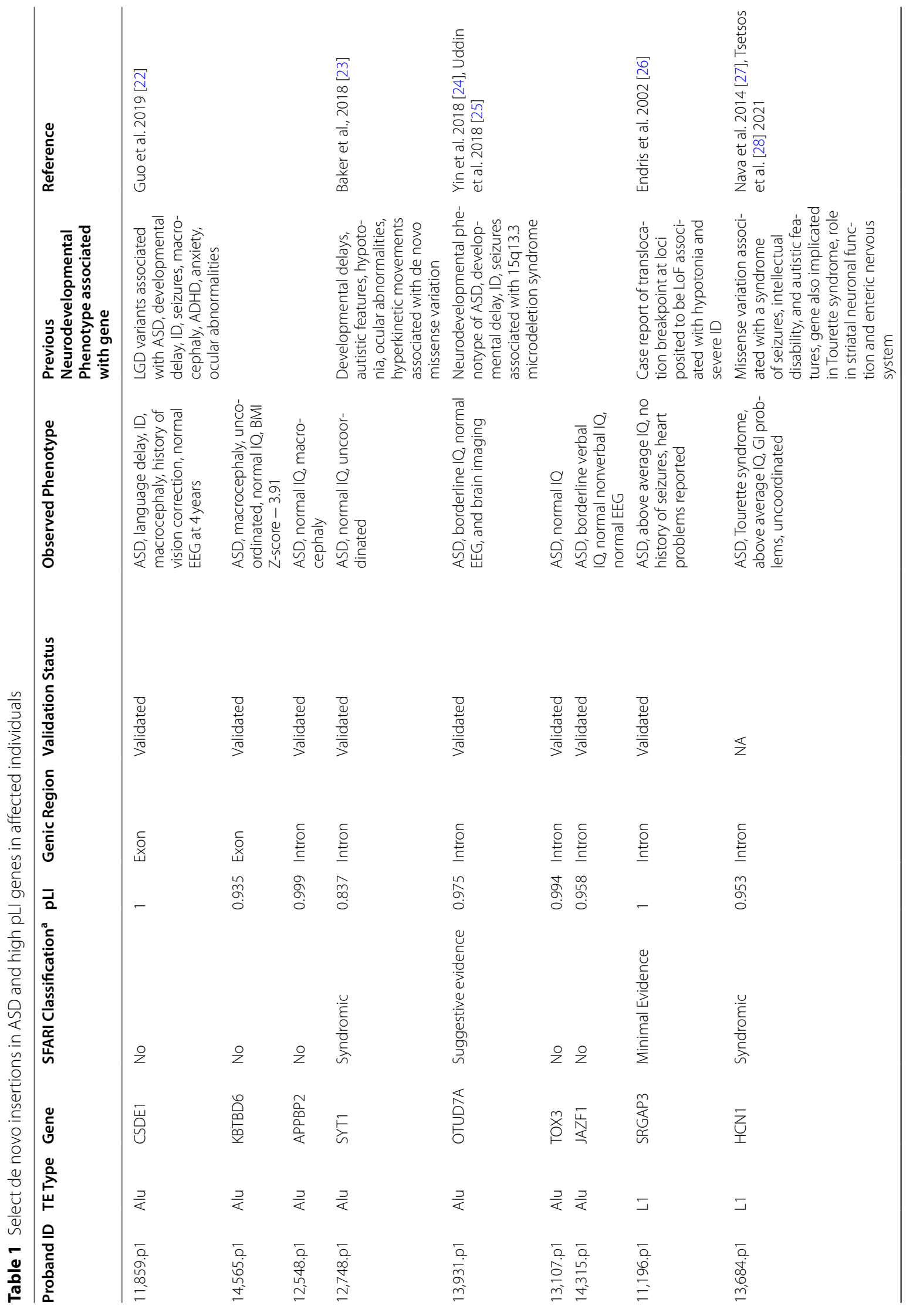




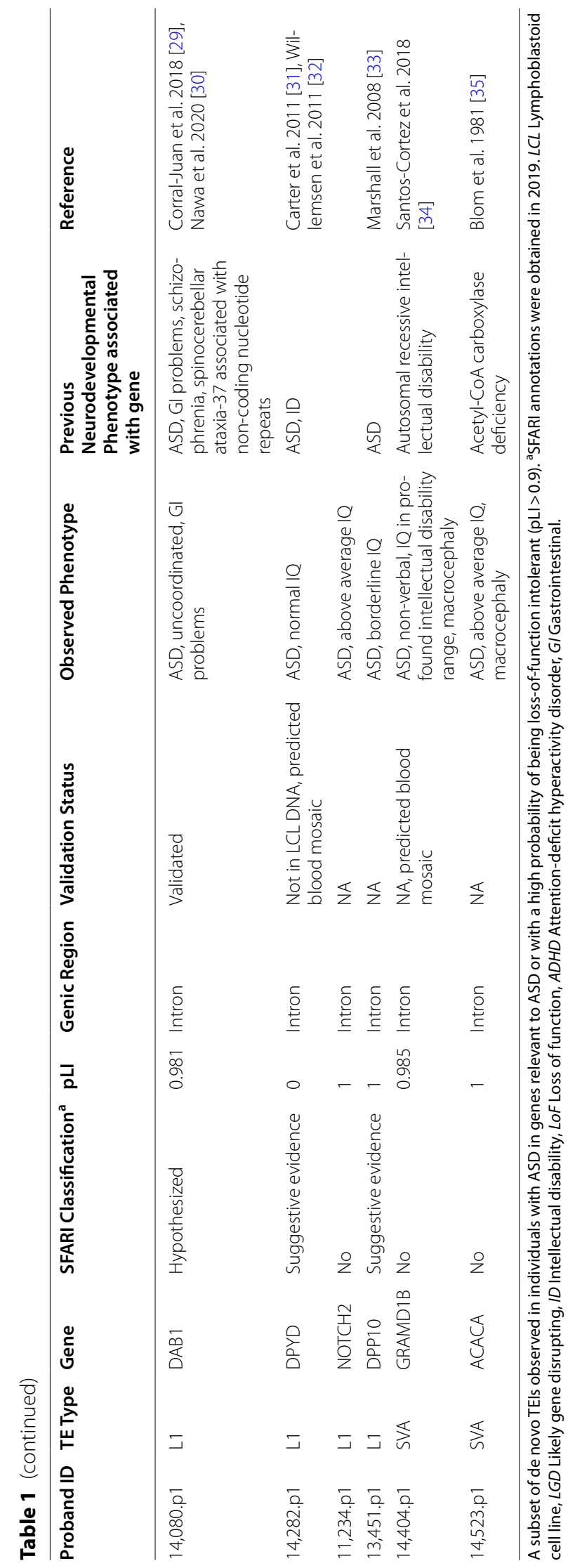




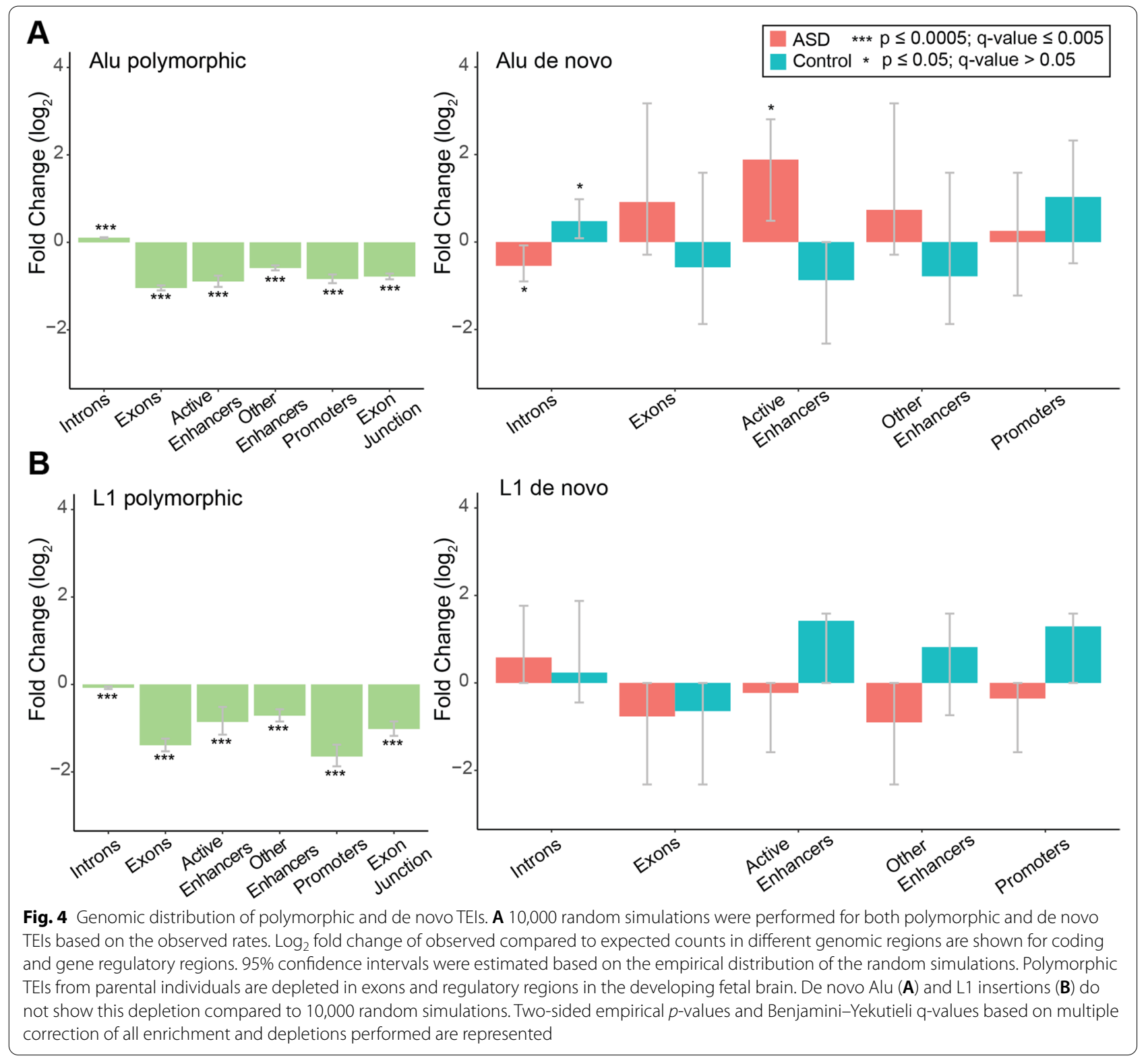

simulating random insertions that consider the L1 endonuclease cleavage site [37, 38] (Additional file 1: Fig. S8). We identified de novo exonic insertions in genes with a high probability of LoF intolerance or haploinsufficiency $(\mathrm{pLI} \geq 0.9)$ [36] only in affected individuals (Table 1 and Additional file 2: Table S2), including an exonic insertion in CSDE1, a gene recently implicated in patients with ASD and neurodevelopmental disabilities [22]. There is a large overlap between SFARI genes and high pLI genes with de novo L1 insertions in cases; $80 \%(4 / 5)$ of SFARI genes with L1 insertions in ASD are also high pLI genes, suggesting that the de novo events can disrupt the haploinsufficient ASD genes and contribute to ASD risk (Table 1).

\section{De novo insertion size distribution resembles polymorphic insertions}

Since paternal and maternal age presents a risk to ASD [39], we tested whether there was a difference in parental age at birth in children with and without de novo TEIs. We found a modest, but not significant, increase in paternal age for children with de novo TEIs compared to those without de novo TEIs $(\mathrm{M}=33.94, \mathrm{SD}=5.63 \mathrm{vs}$. $\mathrm{M}=33.29, \mathrm{SD}=4.71 ; \mathrm{t}(163.42)=1.4452, p=0.1503)$ as 


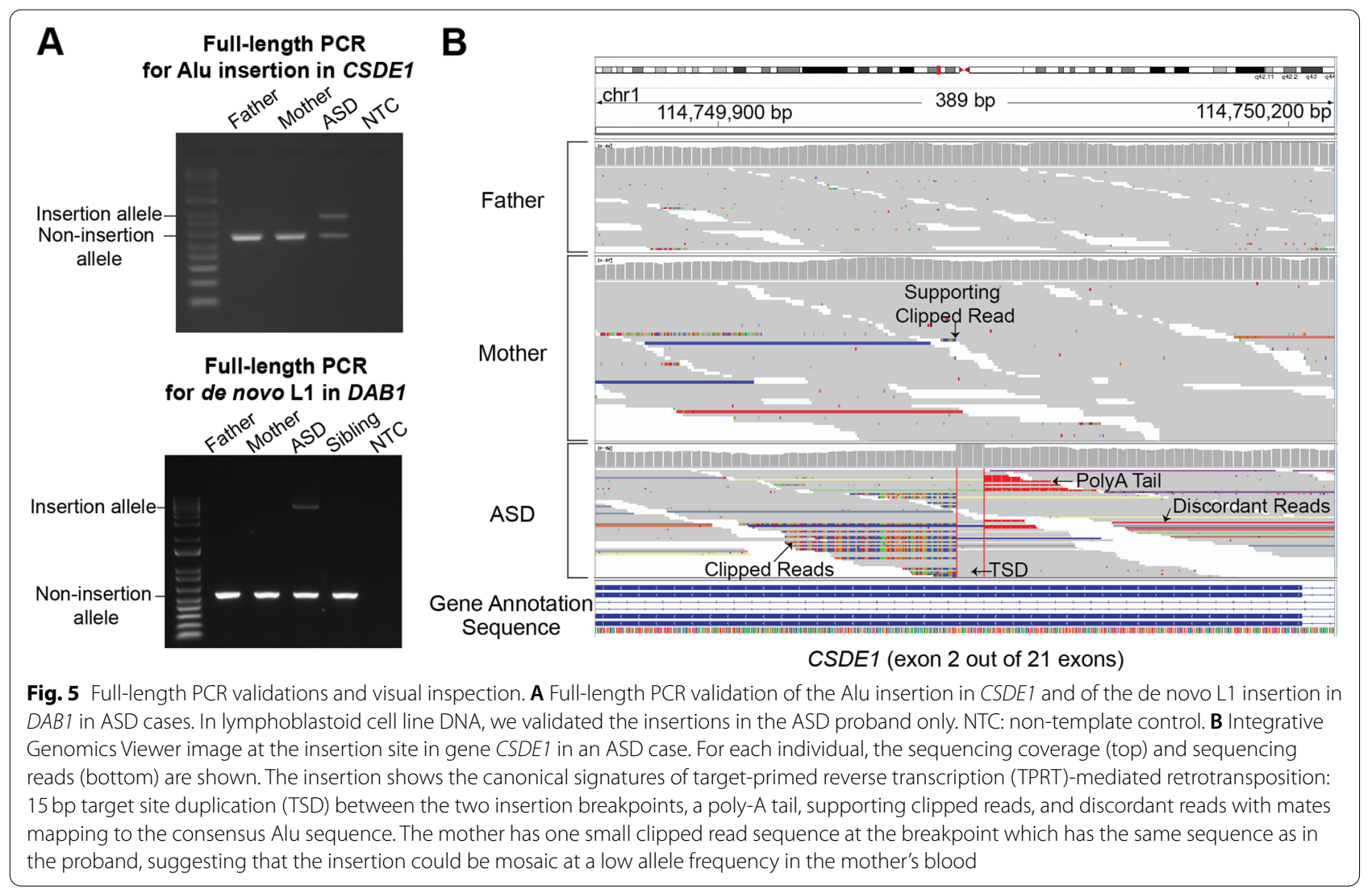

well as increase in maternal age $(\mathrm{M}=31.62, \mathrm{SD}=4.92$ vs. $\mathrm{M}=31.12, \mathrm{SD}=4.92 ; \mathrm{t}(163.75)=1.29, p=0.198$ ) (Additional file 1: Fig. S9). We also estimated the insertion size of polymorphic and de novo TEIs by mapping insertionsupporting reads from $x$ Tea output to TE consensus sequences and obtaining the minimum and maximum mapping coordinates. The distribution of polymorphic L1 insertion size closely resembles previously published data [14] (Additional file 1: Fig. S10A). Overall, de novo TEIs showed similar size distributions to polymorphic TEIs but had different patterns from somatic TEIs, which showed more severe $5^{\prime}$ truncation [17] (Additional file 1: Fig. S10B).

\section{De novo insertions in ASD show an enrichment trend in regulatory regions of the fetal brain}

Some genes with de novo TEIs in ASD are highly expressed in the brain at all stages of development (Additional file 1: Table S4). We found an enrichment of de novo TEIs in ASD in genes upregulated in the prefrontal cortex, although this was not significant after multiple test correction ( $p$-value $=0.0017$, BenjaminiHochberg q-value $=0.07$ ), whereas no such enrichment was detected in controls. Additionally, we found that genes with de novo TEIs were enriched for calciumdependent phospholipid-binding in ASD (adjusted $p$-value $=0.034$ ) but did not find enrichment for any Gene Ontology terms in controls. Several de novo TEIs were also observed in regions with enhancer and promoter chromatin marks in fetal brain development (Additional file 1: Table S5). Thus, we evaluated the enrichment of polymorphic and de novo TEIs in different genomic and epigenomic regions using the Roadmap Epigenomics 25-state model [40]. Polymorphic L1 and Alu insertions were depleted in exons, enhancers, and promoters (Fig. 4; two-sided empirical $p<0.0005$, Benjamini-Yekutieli q-value $<0.0043$ for each category) whereas SVAs did not show a significant depletion in those regions likely due to the limited number of insertions (Additional file 1: Fig. S11 and Table S6). De novo TEIs overall showed patterns within the expected ranges in most regions, however, we observed a trend for more de novo Alu insertions in active enhancer regions in the fetal brain in ASD than expected but not in controls (two-sided empirical $p=0.018$, Benjamini-Yekutieli $q$-value $=0.3$ ). This trend was also observed when considering the L1 endonuclease cleavage site preference in the background model for expected TEIs (Additional 
file 1: Fig. S12). This suggests the intriguing possibility that Alu insertions in neural enhancers might be a rare cause of ASD, though larger samples sizes are needed to test this.

\section{PCR validations confirm ASD relevant retrotransposon insertions}

We selected de novo L1 and Alu insertions from both cases and controls in a subset of ASD and high pLI genes as well as in randomly selected genes for fulllength PCR validation (Additional file 3: Table S7). We validated 22 of 23 (96\%) Alu insertions and 6/7 (86\%) L1 insertions, achieving a high validation rate of $93 \%$ $(28 / 30)$. Validated insertions include a full-length de novo intronic L1 insertion in $D A B 1$, a gene with a high probability of being loss-of-function intolerant $(\mathrm{pLI}=0.981)$ [36] and a hypothesized ASD gene [21, 30] implicated in regulating neuronal migration in development via the Reelin pathway in an isoform dependent manner [41]. We additionally validated an exonic Alu insertion in ASD gene CSDE1 [22] in an ASD proband (Fig. 5A). Our manual IGV inspection identified a single supporting clipped read at the breakpoint (Fig. 5B) in the mother, suggesting that the exonic Alu insertion in CSDE1 could be potentially mosaic at a low allelic fraction in the mother's blood, though low-level contamination from the proband's DNA cannot be completely ruled out. This insertion was fully validated in lymphoblastoid cell line (LCL) DNA in the individual with ASD and was absent in the mother, but LCLs might be expected to be limited in validating low-level mosaic variants (Fig. 5A).

\section{Discussion}

The detection of TEIs in genome sequencing data requires specific pipelines, given their repetitive nature and short read length. These variants have previously been excluded from most routine genetic diagnoses and studies, including for ASD. Furthermore, accurate estimation of de novo TEIs in healthy individuals is important to understand the contribution of de novo TEIs in disease cohorts. Initial methods to determine de novo rates of TEIs relied on indirect methods which compared two reference genomes, making assumptions regarding the time to the most recent common ancestor between human reference genomes [3] and human-chimpanzee divergence time [2]. To directly determine de novo retrotransposition rates, large cohorts are necessary given the infrequency of these events. More recent studies using short-read sequencing technologies have included fewer than 1000 families each, leading to uncertainties in estimates, especially for SVA insertions $[4,10,11]$. They have also not accounted for the lower sensitivity of detection on TEIs using short-read sequencing [12]. Compared to 1 in 20 [2] or 1 in 21 [3] Alu insertions per birth by earlier studies using evolutionary and mutational based methods, our estimate of 1 in 29 births is lower but within the range from more recent work using family genome sequencing data of 1 in 39.7 births (95\% CI 22.4-79.4) [4] (Fig. 2A). L1 rates observed here of 1 in 117 births are also within the ranges observed previously of 1 in 63 births (95\% CI 30.6-153.8) [4] and 1 in 149.2 (95\% CI 72.5-370.4) [10] but higher than the Xing et al. 2009 rate of 1 in 212 births (95\% CI 156-289) [3] (Fig. 2A). Our SVA de novo rates of 1 in 206 births are much higher than the Xing et al. 2009 rate of 1 in 916 births (95\% CI 5031927) [3] but not as high as the Feusier et al. 2019 rate of 1:63 births (95\% CI 30.6-153.8) (Fig. 2A). The large sample size in our study produces more reliable estimates with smaller confidence intervals than previous analyses (Fig. 2A), suggesting that our data provide the most accurate determination of TEI rates up to this time.

Recently published work on the same ASD cohort [12] detected fewer insertions and reported 31\% (1 in $42 \mathrm{Alu}$ ), 49\% (1 in $231 \mathrm{~L} 1$ ), and 33\% ( 1 in 309 SVA) lower de novo insertion rates than ours, possibly due to their exclusion of mosaic insertions in their rate estimates, the use of a less sensitive pipeline [14], and not adjusting for the lower sensitivity for detection of TEIs in short-read data. We detected 47 visually inspected de novo candidates that were not reported by this previous analysis, and within the samples overlapping their cohort and the cohort of this study, we did not detect 27 of their de novo candidates. Only 13 of the 27 undetected events passed our criteria for visual inspection of having two breakpoints, a target site duplication, and a polyA tail while the rest either did not pass these criteria or had been excluded because they were considered inherited events. Most de novo candidates that we missed were not detected because they display few supporting reads, or the parental genomes have clipped reads near the breakpoint.

We demonstrated that the sensitivity and specificity of our pipeline are high (Additional file 1: Fig. S1) through rigorous benchmarking. Despite its comparable performance to MELT, we detected more novel TEIs compared to gnomAD-SV, which uses MELT to detect TEIs. It is likely because the post hoc filtering implemented in the gnomAD-SV study after running MELT [10, 18] reduces the sensitivity but increases the specificity in their analysis, resulting in fewer candidates compared to our analysis. Novel TEIs include candidates with both clipped and discordant read support for one breakpoint and either clipped or discordant read support for the other breakpoint. These "one-and-a-half" sided TEIs may not have a reported TSD but have sufficient read support to be considered 
high-confidence candidates by $x$ Tea (Additional file 1 : Fig. S5A). This group may contain relatively more false positives than insertions with both clipped and discordant read support on both breakpoints as well as a TSD and a polyA tail, i.e., 'two-sided tprt'insertions. We have annotated and included both types of TEIs to maintain high sensitivity and so that we could provide a comprehensive dataset. All the reported candidates in this study have been uploaded to dbVar and users can customize based on the confidence classification rating (Additional files 4, 5, 6, 7, 8 and 9).

Assigning causality to non-coding variants based on clinical phenotypes is challenging, given that most known ASD genes have been discovered in the context of coding LoF variants, yet most individuals with ASD do not have LoF coding variants identified [9]. To understand the clinical phenotypes of individuals with TEIs in high pLI [36] or known ASD genes [21], we reviewed the available clinical data and compared this to any known phenotypes associated with the gene, as well as the scientific literature more generally available (Table 1). Exonic insertions are likely to disrupt the coding sequence and are thus of particular interest. We observed one exonic Alu insertion in CSDE1, which has been recently associated with ASD [22]. The affected proband shared clinical features, albeit non-specific, consistent with the previously described cohort, including ASD, intellectual disability, macrocephaly, and vision impairment. We additionally observed an exonic Alu insertion in KBTBD6 (Table 1). Variation in this gene has not yet been associated with a reported neurodevelopmental phenotype that we are aware of. However, KBTBD6 represents an intriguing candidate gene given its high pLI score $(\mathrm{pLI}=0.935)[36]$ as well as its molecular interactions with known ASD gene CUL3, to mediate the activity of another ASD gene, RAC1 [42]. Studying target genes of exonic de novo TEIs may shed novel biological insight not captured solely with more commonly studied forms of genetic variation in ASD.

We estimated a rate of underlying exonic TEIs of at least 1 in 2288 in ASD, which is similar to the rate of 1 in 2434 cases with developmental disorders reported in a recent exome sequencing study [5]. Although this is lower than other types of de novo genetic drivers of ASD, such as copy number variation, and the contribution of non-coding variants is thought to be smaller than coding LoF variants [10], the strong depletion of polymorphic TEIs in regulatory non-coding regions and enrichment of large de novo L1 insertions $(\sim 6 \mathrm{~kb}$ when full-length) in introns of ASD genes in cases but not in control suggest some of these non-coding events may contribute to ASD risk. Since intronic TEIs can affect gene function through various mechanisms, such as altering RNA expression and splicing [1], TEIs contributing to ASD may present a phenotype different from known phenotypes caused by LoF coding variants or large CNVs in these genes. Including TEIs and structural variants in standard clinical genetic analyses for ASD will continue to expand our knowledge of noncoding variants and could increase the rates of genetic diagnoses.

\section{Conclusions}

We have established $x T e a$, a scalable and sensitive method for detecting TEIs in WGS data on the Amazon cloud platform and applied it to a large cohort of $>8700$ individuals from 2288 ASD quad families. Our work presents important advances in scalable bioinformatic processing of human WGS data and identification of TEIs, which by their nature represent a challenging form of genomic variation to study. We created a catalog of 86,154 polymorphic TEIs, a significant fraction of which were not previously reported. We shared the detailed features of each TEI as a community resource to enhance the understanding of TE biology and population genetics, and to facilitate the identification of disease associated TEIs. By leveraging the large cohort and rigorous pipeline benchmarking, we reported 158 de novo TEIs with robust rate estimates for each TE family. We discovered that although de novo rates between cases and controls are similar, cases had more de novo L1 TEIs in known ASD genes than expected. Most of these TEIs occurred in non-coding genic regions, suggesting that non-coding insertions could have a phenotypic impact. We also detected exonic TEIs in LoF genes in cases, including a causal exonic Alu insertion in CSDE1, known ASD gene [22]. Overall, our analysis suggests a modest, but important, impact of intronic and exonic TE insertions in ASD and implies the existence of diverse TEI-mediated pathogenic mechanisms beyond the insertional mutagenesis of protein-coding sequences. Future work, including both further development of computational methods, as well as experimental functional assessment of the effects and pathogenicity of non-coding TEIs, will be critical in understanding the role of these variants in ASD.

\section{Methods}

\section{Datasets and data processing with $x T e a$}

Whole-genome data from the SSC from phases: Pilot, Phase 1, Phase 2, Phase 3-1, Phase 3-1, and Phase 4 were analyzed. The analyzed data consists of ASD families with one affected individual, two unaffected parents, and for 1860 of these families, one unaffected sibling was analyzed as the unaffected control. To process this massive amount of $>9000$ individual whole genomes, we 
optimized for scalability a TEI detection computational tool, xTea [13] (https://github.com/parklab/xTea) and implemented a dockerized version on Amazon Web Services (Additional file 1: Table S8). After removing outlier results and confirming that these were due to corrupted bam files with incomplete sequences or failed $x$ Tea runs, we analyzed WGS data from $\sim 2288$ ASD affected individuals and 1856 unaffected siblings with both parents sequenced (Additional file 1: Table S1 and Table S3 for sample sizes per TE type). The approximate average sequencing depth, as determined by $x$ Tea, was $39.4 \mathrm{x}$. Paired-end reads were 151 base pairs in length.

\section{TEl identification with $x T e a$}

For each cram file, $x$ Te $a$ ran three major steps to call TE insertions. First, raw candidate sites were collected based on whether there were enough qualified clipped reads at the breakpoints, where part of the read is aligned to the flanking region while the clipped part is well aligned to the consensus TE sequence. Second, for each passed candidate site we checked whether there was enough discordant reads support. Here, we consider a pair of reads with one read aligned to the flanking region and its mate aligned to the TE consensus sequence or other copies as discordant. Third, we ran TE-type specific filters to reduce false positives in both polymorphic and de novo insertions (see Additional file 1). xTea candidates were classified as "high" or "low" confidence insertions depending on whether enough insertion supporting features were distributed on both sides of the breakpoint. We only included insertions classified as "high confidence".

\section{Annotation of non-redundant polymorphic TEls}

After obtaining the $x T e a$ high confidence insertions for each individual, we excluded calls where the clipped and discordant reads mapped above the consensus size $x$ Tea uses for mapping for AluY, L1HS, and SVA (282, 6120, and 1400 base pairs respectively). This removed some Alu insertions, which tended to be polyA expansion artifacts. Since breakpoint positions can have slight differences between individuals, these insertions were given a 40 base pair margin from the midpoint of the breakpoints and were merged if they overlapped to obtain a unique set of non-redundant TEIs in the SSC cohort.

To determine whether insertions in the SSC cohort were known or novel, merged TEI calls were overlapped with the breakpoints from gnomAD [18], 1000 genomes [14], or a compilation of other studies obtained from Evrony et al. [16] to obtain insertions in our cohort that are not found in these studies (novel), known TEIs which overlap, as well as known TEIs which overlap to individual studies only.

TSD sizes were obtained from the $x$ Tea output. The median size was selected for the merged insertions obtained with a 40 base-pair margin as described above. De novo candidates were analyzed separately. We also analyzed high-confidence TEIs classified by $x T e a$ as "twosided target-primed reverse transcription (TPRT)". These candidates are resolved on both breakpoints and have reads supporting a TSD and a polyA tail.

\section{Evaluating the performance of $x T e a$}

We created a haplotype-resolved dataset of non-reference TEIs in the Genome in a Bottle sample NA24385/ HG002 [43] that has been sequenced with both long and short-read technologies. We evaluated the performance of xTea [13], MELT [14], and Mobster [15] using this dataset as the benchmark as described previously [13]. Briefly, we tested the sensitivity and specificity of these tools for detecting 1642 (1355 Alu, 197 L1, and 90 SVA) high-confidence TEIs detected in the NA24385/HG002 genome but not in the reference genome in pair-end downsampled Illumina WGS data. Note that the xTea version used in this study is different from the latest version reported in the xTea method paper [13].

\section{Calculation of TEI population allele frequency}

The merged insertions were genotyped with the $x T e a$ genotyping module which uses a random forest model to genotype TEIs. We trained this model using 14 features from high confidence TEIs obtained from a subset of 1800 unaffected trio families from this cohort, as described previously [13]. If an insertion was detected in an unaffected child and only in one parent, and the other parent did not have any supporting clipped or discordant reads, it was labeled as heterozygous (0/1); if an insertion detected in a child did not have any supporting reads in the parental genomes, it was considered a false positive and labeled as reference homozygous ( $0 / 0)$; if an insertion was detected in both parents, had a ratio of discordant $/$ (discordant + concordant $)>0.85$, and no fully mapped reads at the breakpoint, it was labeled as homozygous (1/1). $70 \%$ of the insertion sites were used for training, and the accuracy in the remaining $30 \%$ of the sites was $99.7 \%$. The population allele frequency (PAF) was calculated using only parental genomes in the cohort, which were unaffected and unrelated. Specifically, PAF for each polymorphic TEI was defined as the number of alleles carrying the TEI in the parents divided by the total number of chromosomes in the population (i.e., $2 \times$ the number of parents). 


\section{Identification and rate estimation of de novo TEls}

To detect de novo insertions, we selected calls that did not have supporting reads in parental raw xTea output files and were confirmed via manual inspection. We further excluded de novo candidates which overlapped with reference and KNR TEIs (see Additional file 1). De novo retrotransposition rates were calculated as the number of de novo TEIs for both ASD affected and unaffected siblings divided by the total sample size. Samples that failed the $x T e a$ run were excluded from the analysis, resulting in a sample size of $n=4142$ for L1, $n=4143$ for Alu, and $n=4148$ for SVA (Additional file 1: Table S3). Rates and confidence intervals from previous studies were obtained from Feusier et al. [4]. The 95\% confidence intervals for de novo rates in the SSC cohort were obtained in the same manner, with an exact binomial confidence interval estimate.

We adjusted the observed de novo rates to account for sensitivity loss in short-read sequencing data and to obtain precise estimates. Specifically, we measured $x T e a$ sensitivity on the downsampled (39.4x) Illumina genome sequencing data from HG002, the HapMap sample extensively profiled by multiple sequencing platforms by the Genome in a Bottle consortium [43] using a highquality catalog of haplotype-resolved non-reference TEIs for the sample (see Additional file 1 and Additional file 1: Fig. S1). We adjusted the number of total ASD and control de novo insertions by dividing the observed rate by the sensitivity for heterozygous TEIs, after filtering young reference and KNR TEIs, and obtained an exact binomial 95\% confidence interval.

\section{Enrichment analysis using simulated TEls}

We performed simulations to calculate the probability of the number of observed insertions in ASD genes, high pLI $(\mathrm{pLI} \geq 0.9)$ genes, or in different genomic regions occurring by chance (see Additional file 1). Using the observed de novo TEIs candidates in ASD and unaffected siblings and the number of unique polymorphic insertions in parents, we simulated the same number of insertions of the same size in random regions of the genome. We also performed these simulations considering the L1 endonuclease cleavage site preference, as described previously $[37,38]$. Here, used the code provided by Wildschutte et al. (https:// github.com/KiddLab/random-sample-by-ppm) to simulate random insertions using a position probability matrix corresponding to the L1 cleavage sites from HeLa cells [38]. We excluded the same young reference TE regions and KNR regions we excluded when detecting de novo TEIs for de novo simulations and excluding young reference TE regions for polymorphic insertions for both simulations. We performed 10,000 simulations and determined the number of random insertions that overlapped a SFARI gene, high pLI gene, or region of interest per simulation. We determined if the observed value fell on the upper or lower end of the observed distribution, with a pseudo count of 1 , to obtain a $p$-value. For example, the upper $p$-value is defined as $(r+1) /(n+1)$, where $r$ is the number of simulations greater or equal to the observed value and $n$ is the number of simulations. This value was multiplied by 2 for an empirical two-sided $p$-value. 95\% CIs were calculated by obtaining the 0.025 and 0.975 percentiles of the null distribution. The $\log _{2}$ FC was calculated as the $\log _{2}$ (observed value/mean of the null distribution) and the $95 \%$ CIs are plotted as their $\log _{2}$ values. These $p$-values were corrected for multiple testing with the Benjamini \& Yekutieli method, to account for dependency between tests.

\section{Gene list enrichment in genes with TEls}

We tested whether genes with TEIs in ASD or controls were enriched for genes overexpressed in tissues in the Human Gene Atlas list using Enrichr [44]. We also tested for enrichment of gene ontology terms in the subset of genes with TEIs using g:Profiler [45] in only annotated genes, with a user threshold of 0.05 and a significant threshold for multiple testing correction with the g:SCS threshold method.

\section{PCR validations}

Twenty four cases were chosen for full-length PCR validation based on their clinical relevance by selecting variants that occurred in SFARI, high pLI, or brain expressed genes and 13 cases were randomly selected for a total of $12 \mathrm{~L} 1$ insertions and $25 \mathrm{Alu}$ insertions (Additional file 3: Table S7). We developed a pipeline for designing specific primers and tested and optimized the PCR protocols for each primer pair in control DNA before validating them in the SSC samples (Additional file 1). Out of $12 \mathrm{~L} 1$ primer pairs designed for validations of de novo insertions, we were able to optimize 9 primer pairs, and we optimized 23 Alu primer pairs out of 25. Two of the L1 primers were selected for mosaic candidates in 1 case and 1 control and were considered separately for validation rates. Validations were performed with $20 \mathrm{ng}$ of DNA from each available family member from lymphoblastoid cell lines which were provided by the Rutgers University Cell and DNA Repository. This was done by confirming the presence of both an insertion and a non-insertion allele band near or at the expected insertion size in the samples with predicted insertions, and only a non-insertion allele band in the other family members. Water was used instead of DNA as a non-template control for each primer pair. 


\section{Supplementary Information}

The online version contains supplementary material available at https://doi. org/10.1186/s13100-021-00256-w.

Additional file 1: Supplementary Methods, Figure S1. The version of xTea used in this study has a high performance and is comparable to MELT in short-read Illumina WGS data. Figure S2. Polymorphic and de novo transposable element insertions (TEIs) in the SSC cohort. Figure S3. The number of Alu TEls detected per individual is different for some populations. Figure S4. Percentage of insertions which were not found in previous studies (novel) or overlap with TEls from previous analyses (known). Figure S5. Target site duplication (TSD) size distribution for de novo and polymorphic novel and known non-reference (KNR) TEls. Figure S6. Comparison of population allele frequencies (PAFs) between unrelated parental individuals in the SSC cohort and gnomAD-SVTEIs. Figure S7. The observed number of de novo TEls in high probability of being loss of function intolerant (pLI) genes compared to expected TEls based on 10,000 random simulations. Figure S8. The observed number of de novo TEls in SFARI ASD genes and high pLI genes compared to expected TEls based on 10,000 random simulations using a position probability matrix to consider the L1 endonuclease cleavage site. Figure S9. Parental age at birth of children with and without TEls for cases and controls combined. Figure S10. Estimated insertion size of TEls. Figure S11. Enrichment and depletion of TEls in coding and gene regulatory regions. Figure S12. Enrichment and depletion of TEls in coding and gene regulatory regions. Table S1. Polymorphic insertions sample sizes. Table S3. De novo insertion rates and sample sizes. Table $\mathbf{S 4}$. De novo insertions that overlap the top $10 \%$ expressed genes in the neocortex during development. Table S5. Number of de novo insertions overlapping regions with epigenetic annotation in fetal brain. Table S6. Number of observed polymorphic insertions in parental SSC samples overlapping regions with epigenetic annotation in fetal brain. Table $\mathbf{S 8}$. Memory and time cost of xTea on different numbers of CPU cores.

Additional file 2: Table S2: De novo insertions in ASD and controls.

Additional file 3: Table S7: PCR validations primers and samples.

Additional file 4. Alu polymorphic and de novo TEls for the entire cohort intervals after merging with a 40 base-pair margin.

Additional file 5. L1 polymorphic and de novo TEls for the entire cohort intervals after merging with a 40 base-pair margin.

Additional file 6. SVA polymorphic and de novo TEls for the entire cohort intervals after merging with a 40 base-pair margin.

Additional file 7. Alu polymorphic and de novo TEls for unrelated parents in the cohort after merging with a 40 base-pair margin.

Additional file 8. L1 polymorphic and de novo TEls for unrelated parents in the cohort after merging with a 40 base-pair margin.

Additional file 9. SVA polymorphic and de novo TEls for unrelated parents in the cohort after merging with a 40 base-pair margin.

\section{Acknowledgments}

We sincerely thank the families who participated in this research in the Simons Foundation Autism Research Initiative (SFARI) and Simons Simplex Collection (SSC). We acknowledge the clinicians who contributed to the collection of samples and phenotypic data, the SSC principal investigators, and the SFARI staff, in particular, S. Xiao and R. Rana for providing additional information. We thank Rutger's RUCDR Infinite Biologics for providing DNA samples. We are grateful to S. Hill and J. Neil for their help accessing the SSC data, and to M.E. Talkowski, P.R. Loh, E. Macosko, B. Zhao, G.D. Evrony, and R.E Andersen for their advice.

\section{Authors' contributions}

Conceptualization: C.A.W., E.A.L., R.B.M.; Data Curation: R.B.M.; Formal Analysis: R.B.M., C.C.; Y.G., T.S., J.C.; Funding acquisition: E.A.L., C.A.W.; Investigation: R.B.M., C.D., Y.G., T.S.; Methodology: R.B.M., C.C.; Project administration: E.A.L, R.B.M.; Resources: C.C., J.C.; Software: C.C., R.B.M., S.L., J.C.; Supervision: E.A.L., C.A.W., P.J.P.; Validation: R.B.M; Visualization: R.B.M; Writing - original draft:
R.B.M; Writing - review \& editing: E.A.L., C.A.W., C.D., C.C. All authors read and approved the final manuscript.

\section{Funding}

This work was supported by the National Institutes of Health Cloud Credits Model Pilot, part of the National Institutes of Health Big Data to Knowledge program; the Fundacion Mexico en Harvard and Consejo Nacional de Ciencia yTecnología [to R.B.M.]; the Translational Post-doctoral Training in Neurodevelopment Program and National Institute of Mental Health (https://www. nimh.nih.gov/index.shtml) [grant number T32MH1 12510 to C.D.]; the National Institutes of Health (https://www.nih.gov/) [grant numbers K01 AG051791 to E.A.L., DP2 AG072437 to E.A.L.]; the Suh Kyungbae Foundation [to E.A.L.], the Charles H. Hood Foundation (http://charleshoodfoundation.org/) [to E.A.L.]; the National Institute of Mental Health through the Brain Somatic Mosaicism Network (https://bsmn.synapse.org/index.html) [grant number U01MH106883 to C.A.W.]; the Allen Frontiers Program through the Allen Discovery Center for Human Brain Evolution (https://brainevolution.org/) [to E.A.L and C.A.W.], the Hock E. Tan and K. Lisa Yang Center for Autism Research at Harvard University (https://mcgovern.mit.edu/centers/tan-yang-center/) [to C.A.W.], and the Howard Hughes Medical Institute (https://www.hhmi.org/) [to C.A.W.]. The funders had no role in study design, data collection, and analysis, decision to publish, or preparation of the manuscript.

\section{Availability of data and materials}

The parental high-confidence TEls from our study are available in the dbVar repository under the study accession "nstd203", https://www.ncbi.nlm.nih.gov/ dbvar/?term=nstd203. A vcf of all TEls identified with $x$ Tea and their annotations can be found here: https://ftp.ncbi.n/m.nih.gov/pub/dbVar/data/Homo_ sapiens/by_study/genotype/nstd203. We also created two merged TEl sets from all the TEls reported in the vcf file-one from all SSC individuals and the other only from parental genomes_for each TE family. The TEl sets along with TSD size, estimated insertion size, genotype, and confidence classifications are available as supplementary material (Additional files 4, 5, 6, 7, 8 and 9). A 40-bp margin was used to merge TEls since the predicted breakpoints can slightly differ even for the same TEl depending on the supporting clipped reads in each sample, resulting in the 86,154 reported insertions.

The most current version of $x T e a$, including the filtering and genotyping module used in this study after running the initial pipeline, can be found here: https://github.com/parklab/xTea/. The docker file and version (warbler/ xteab:v9) used in our analysis can be found here: https://hub.docker.com/ repository/docker/warbler/xteab and the GitHub xTea branch used is located here: https://github.com/parklab/xTea/tree/release_xTea_cloud_1.0.0-beta. This software is platform-independent and the programming language is Python. The license and additional requirements can be found here: https:// github.com/parklab/xTea/. The code used for detection of de novo insertions and for designing primers for full-length validation of TEls is located here: https://github.com/ealeelab/TEs_ASD.

The raw sequencing data that support the findings of this study are available from the Simons Foundation Autism Research Initiative, but restrictions apply to the availability of these data and so are not publicly available. Access may be requested through https://base.sfari.org/.

\section{Declarations}

Ethics approval and consent to participate

This research was performed following a protocol approved by the institutional review board of the Boston Children's Hospital. Only de-identified data and samples were used following approval and receipt from the Simons Foundation Autism Research Initiative.

\section{Consent for publication}

Not applicable.

\section{Competing interests}

The authors declare that they have no competing interests.

\section{Author details}

${ }^{1}$ Division of Genetics and Genomics, Manton Center for Orphan Disease, Boston Children's Hospital, Boston, MA, USA. ${ }^{2}$ Broad Institute of MIT and Harvard, Cambridge, MA 02142, USA. ${ }^{3}$ Department of Biomedical Informatics, Harvard 
Medical School, Boston, MA, USA. ${ }^{4}$ Division of Developmental Medicine, Boston Children's Hospital, Harvard Medical School, Boston, MA, USA. ${ }^{5}$ Department of Genetics, Harvard Medical School, MA, Boston, USA. ${ }^{6}$ Department of Pediatrics, Harvard Medical School, MA, Boston, USA. ${ }^{7}$ Department of Neurology, Harvard Medical School, Boston, MA, USA. ${ }^{8}$ Howard Hughes Medical Institute, Boston Children's Hospital, Boston, MA, USA.

Received: 23 April 2021 Accepted: 2 November 2021

Published online: 27 November 2021

\section{References}

1. Hancks DC, Kazazian HH Jr. Roles for retrotransposon insertions in human disease. Mob DNA. 2016;7:9.

2. Cordaux R, Hedges DJ, Herke SW, Batzer MA. Estimating the retrotransposition rate of human Alu elements. Gene. 2006;373(Supplement C):134-7

3. Xing J, Zhang Y, Han K, Salem AH, Sen SK, Huff CD, et al. Mobile elements create structural variation: analysis of a complete human genome. Genome Res. 2009;19(9):1516-26.

4. Feusier J, Watkins WS, Thomas J, Farrell A, Witherspoon DJ, Baird L, et al. Pedigree-based estimation of human mobile element retrotransposition rates. Genome Res. 2019:29(10):1567-77.

5. Gardner EJ, Prigmore E, Gallone G, Danecek P, Samocha KE, Handsaker $J$, et al. Contribution of retrotransposition to developmental disorders. Nat Commun. 2019;10(1):4630.

6. Kim J, Hu C, Moufawad El Achkar C, Black LE, Douville J, Larson A, et al. Patient-customized oligonucleotide therapy for a rare genetic disease. N Engl J Med. 2019;381(17):1644-52.

7. American Psychiatric Association. Diagnostic and statistical manual of mental disorders : DSM-5. 5th ed. Washington, DC: American Psychiatric Publishing; 2013. p. xliv. 947 pages $p$

8. lakoucheva LM, Muotri AR, Sebat J. Getting to the cores of autism. Cell. 2019:178(6):1287-98

9. Iossifov I, O'Roak BJ, Sanders SJ, Ronemus M, Krumm N, Levy D, et al. The contribution of de novo coding mutations to autism spectrum disorder. Nature. 2014;515(7526):216-21.

10. Werling DM, Brand H, An JY, Stone MR, Zhu L, Glessner JT, et al. An analytical framework for whole-genome sequence association studies and its implications for autism spectrum disorder. Nat Genet. 2018;50(5):727-36.

11. Brandler WM, Antaki D, Gujral M, Kleiber ML, Whitney J, Maile MS, et al. Paternally inherited cis-regulatory structural variants are associated with autism. Science. 2018;360(6386):327-31.

12. Belyeu JR, Brand H, Wang H, Zhao X, Pedersen BS, Feusier J, et al. De novo structural mutation rates and gamete-of-origin biases revealed through genome sequencing of 2,396 families. Am J Hum Genet. 2021;108(4):597-607

13. Chu C, Borges-Monroy R, Viswanadham VV, Lee S, Li H, Lee EA, et al. Comprehensive identification of transposable element insertions using multiple sequencing technologies. Nat Commun. 2021;12(1):3836.

14. Gardner EJ, Lam VK, Harris DN, Chuang NT, Scott EC, Pittard WS, et al. The Mobile element locator tool (MELT): population-scale mobile element discovery and biology. Genome Res. 2017;27(11):1916-29.

15. Thung DT, de Ligt J, Vissers LE, Steehouwer M, Kroon M, de Vries P, et al. Mobster: accurate detection of mobile element insertions in next generation sequencing data. Genome Biol. 2014;15(10):488.

16. Evrony GD, Lee E, Mehta BK, Benjamini Y, Johnson RM, Cai X, et al. Cell lineage analysis in human brain using endogenous retroelements. Neuron. 2015;85(1):49-59.

17. Lee $E$, Iskow R, Yang L, Gokcumen O, Haseley P, Luquette LJ 3rd, et al. Landscape of somatic retrotransposition in human cancers. Science. 2012;337(6097):967-71

18. Collins RL, Brand H, Karczewski KJ, Zhao X, Alfoldi J, Francioli LC, et al. A structural variation reference for medical and population genetics. Nature. 2020;581(7809):444-51.

19. Zhou W, Emery SB, Flasch DA, Wang Y, Kwan KY, Kidd JM, et al. Identification and characterization of occult human-specific LINE-1 insertions using long-read sequencing technology. Nucleic Acids Res. 2020;48(3):1146-63.

20. Ewing AD, Smits N, Sanchez-Luque FJ, Faivre J, Brennan PM, Richardson SR, et al. Nanopore sequencing enables comprehensive transposable element epigenomic profiling. Mol Cell. 2020;80(5):915-28 e5.

21. Abrahams BS, Arking DE, Campbell DB, Mefford HC, Morrow EM, Weiss LA, et al. SFARI gene 2.0: a community-driven knowledgebase for the autism spectrum disorders (ASDs). Mol Autism. 2013;4(1):36.

22. Guo H, Li Y, Shen L, Wang T, Jia X, Liu L, et al. Disruptive variants of CSDE1 associate with autism and interfere with neuronal development and synaptic transmission. Sci Adv. 2019;5(9):eaax2166.

23. Baker K, Gordon SL, Melland H, Bumbak F, Scott DJ, Jiang TJ, et al. SYT1-associated neurodevelopmental disorder: a case series. Brain. 2018;141(9):2576-91.

24. Yin J, Chen W, Chao ES, Soriano S, Wang L, Wang W, et al. Otud7a knockout mice recapitulate many neurological features of $15 q 13.3$ microdeletion syndrome. Am J Hum Genet. 2018;102(2):296-308.

25. Uddin M, Unda BK, Kwan V, Holzapfel NT, White SH, Chalil L, et al. OTUD7A regulates neurodevelopmental phenotypes in the $15 q 13.3$ microdeletion syndrome. Am J Hum Genet. 2018:102(2):278-95.

26. Endris V, Wogatzky B, Leimer U, Bartsch D, Zatyka M, Latif F, et al. The nove rho-GTPase activating gene MEGAP/ srGAP3 has a putative role in severe mental retardation. Proc Natl Acad Sci U S A. 2002;99(18):11754-9.

27. Nava C, Dalle C, Rastetter A, Striano P, de Kovel CG, Nabbout R, et al. De novo mutations in HCN1 cause early infantile epileptic encephalopathy. Nat Genet. 2014;46(6):640-5.

28. Tsetsos F, Yu D, Sul JH, Huang AY, IIImann C, Osiecki L, et al. Synaptic processes and immune-related pathways implicated in Tourette syndrome. Transl Psychiatry. 2021;11(1):56.

29. Corral-Juan M, Serrano-Munuera C, Rabano A, Cota-Gonzalez D, SegarraRoca A, Ispierto L, et al. Clinical, genetic and neuropathological characterization of spinocerebellar ataxia type 37. Brain. 2018;141(7):1981-97.

30. Nawa Y, Kimura H, Mori D, Kato H, Toyama M, Furuta S, et al. Rare singlenucleotide DAB1 variants and their contribution to schizophrenia and autism spectrum disorder susceptibility. Hum Genome Var. 2020;7(1):37.

31. Carter MT, Nikkel SM, Fernandez BA, Marshall CR, Noor A, Lionel AC, et al. Hemizygous deletions on chromosome 1p21.3 involving the DPYD gene in individuals with autism spectrum disorder. Clin Genet. 2011;80(5):435-43.

32. Willemsen MH, Valles A, Kirkels LA, Mastebroek M, Olde Loohuis N, Kos A, et al. Chromosome 1p21.3 microdeletions comprising DPYD and MIR137 are associated with intellectual disability. J Med Genet. 2011:48(12):810-8.

33. Marshall CR, Noor A, Vincent JB, Lionel AC, Feuk L, Skaug J, et al. Structural variation of chromosomes in autism spectrum disorder. Am J Hum Genet. 2008;82(2):477-88

34. Santos-Cortez RLP, Khan V, Khan FS, Mughal ZU, Chakchouk I, Lee K, et al. Novel candidate genes and variants underlying autosomal recessive neurodevelopmental disorders with intellectual disability. Hum Genet. 2018;137(9):735-52.

35. Blom W, de Muinck Keizer SM, Scholte HR. Acetyl-CoA carboxylase deficiency: an inborn error of de novo fatty acid synthesis. N Engl J Med. 1981;305(8):465-6.

36. Lek M, Karczewski KJ, Minikel EV, Samocha KE, Banks E, Fennell T, et al. Analysis of protein-coding genetic variation in 60,706 humans. Nature. 2016:536(7616):285-91.

37. Wildschutte JH, Baron A, Diroff NM, Kidd JM. Discovery and characterization of Alu repeat sequences via precise local read assembly. Nucleic Acids Res. 2015:43(21):10292-307.

38. Gilbert N, Lutz S, Morrish TA, Moran JV. Multiple fates of L1 retrotransposition intermediates in cultured human cells. Mol Cell Biol. 2005;25(17):7780-95.

39. Croen LA, Najjar DV, Fireman B, Grether JK. Maternal and paternal age and risk of autism spectrum disorders. Arch Pediatr Adolesc Med. 2007:161(4):334-40.

40. Roadmap Epigenomics C, Kundaje A, Meuleman W, Ernst J, Bilenky M, Yen A, et al. Integrative analysis of 111 reference human epigenomes. Nature. 2015;518(7539):317-30

41. Gao Z, Godbout R. Reelin-Disabled-1 signaling in neuronal migration: splicing takes the stage. Cell Mol Life Sci. 2013;70(13):2319-29. 
42. Genau HM, Huber J, Baschieri F, Akutsu M, Dotsch V, Farhan H, et al. CUL3KBTBD6/KBTBD7 ubiquitin ligase cooperates with GABARAP proteins to spatially restrict TIAM1-RAC1 signaling. Mol Cell. 2015;57(6):995-1010.

43. Zook JM, Catoe D, McDaniel J, Vang L, Spies N, Sidow A, et al. Extensive sequencing of seven human genomes to characterize benchmark reference materials. Sci Data. 2016;3:160025.

44. Chen EY, Tan CM, Kou Y, Duan Q, Wang Z, Meirelles GV, et al. Enrichr: interactive and collaborative HTML5 gene list enrichment analysis tool. BMC Bioinformatics. 2013;14:128.

45. Raudvere U, Kolberg L, Kuzmin I, Arak T, Adler P, Peterson H, et al. G:profiler: a web server for functional enrichment analysis and conversions of gene lists (2019 update). Nucleic Acids Res. 2019;47(W1):W191-W8

\section{Publisher's Note}

Springer Nature remains neutral with regard to jurisdictional claims in published maps and institutional affiliations.

- fast, convenient online submission

- thorough peer review by experienced researchers in your field

- rapid publication on acceptance

- support for research data, including large and complex data types

- gold Open Access which fosters wider collaboration and increased citations

- maximum visibility for your research: over 100M website views per year

At BMC, research is always in progress.

Learn more biomedcentral.com/submissions 